\title{
A DECOMPOSITION THEORY FOR UNITARY REPRESENTA- TIONS OF LOCALLY COMPACT GROUPS
}

BY JOHN ERNEST ${ }^{1}$

Communicated by Deane Montgomery, March 27, 1961

The purpose of this note is to announce a decomposition theory for unitary representations over a separable complex Hilbert space, prototyped after the type I theory given in [6], but which applies to all separable locally compact groups. The theory uses the central (or canonical) decomposition throughout and the "building blocks" are the primary (or factor) representations. (A representation is primary if it cannot be expressed as the direct sum of two disjoint representations. Two representations $L$ and $M$ are disjoint, denoted $L \circ M$, if no subrepresentation of one is equivalent to any subrepresentation of the other.)

Proposition 1. Let $L$ denote a representation of a separable locally compact group $G$ with a decomposition $L \simeq \int_{Z} L^{y} d \mu(y)$ over a separable Borel space $Z$, such that the range of the corresponding projection-valued measure is contained in the center of the commuting algebra, $R(L, L)$, of $L$. Then there exists a Borel subset $Z^{\prime}$ of $Z$ such that $\mu\left(Z-Z^{\prime}\right)=0$ and $L^{y} \delta L^{y^{\prime}}$ whenever $y, y^{\prime} \in Z^{\prime}$ and $y \neq y^{\prime}$.

This result has also been announced by Naimark [9, Theorem 1] in the important special case where the decomposition is the central decomposition. The proof of Proposition 1 is a simple perturbation of a proof given by Guichardet [3] for the special case of the central decomposition of a multiplicity free representation.

A representation $L$ covers a representation $M$, denoted $L\} M$, if no subrepresentation of $M$ is disjoint from $L . L$ and $M$ are said to be quasi-equivalent, denoted $L \sim M$, if $L$ covers $M$ and $M$ covers $L$.

Proposition 2. Two representations $L$ and $M$ are quasi-equivalent if and only if they have central decompositions over the same measure space, say $L \simeq \int_{Z} L^{y} d \mu(y)$ and $M \simeq \int_{Z} M^{y} d \mu(y)$, such that $L^{y} \sim M^{y}$ for $\mu$-almost all $y$.

The proof of Proposition 2 is an adaptation of known facts about direct integrals of isomorphisms of von Neumann algebras. (Cf. [1].)

The collection $Q$ of all quasi-equivalence classes of representations of a group $G$ (always assumed to be separable locally compact), partially ordered by the covering relation defined above, is a distribu-

\footnotetext{
1 The author is a National Science Foundation Fellow.
} 
tive $\sigma$-lattice in which one may take relative complements. In our theory the dual object, denoted $\widetilde{G}$, is taken to be the collection of minimal elements of $Q$ or, equivalently, the collection of all quasiequivalence classes of primary representations of $G$. We call $\widetilde{G}$ the quasi-dual to distinguish it from the traditional dual, $\hat{G}$, the collection of unitary equivalence classes of irreducible representations of $G$.

$\tilde{G}$ is given a Borel structure as follows. Define $G^{c}$, the "concrete" representations of $G$, and a Borel structure for $G^{c}$ as in $\S 9$ of [6]. Let $G^{p}$ denote the subset of $G^{c}$ consisting of primary representations. If $B$ is a Borel subset of $G^{c}$, let $B^{a}$ denote the subset of elements in $G^{o}$ which are quasi-equivalent to some element in $B$. Give $G^{p}$ the smallest Borel structure containing all sets $B$ and $B^{q}$ such that $B$ is a Borel subset of $G^{c}$ for which $B \subset G^{p}$. Give $\widetilde{G}$ the Borel structure defined as a quotient space of $G^{p}$. (Cf. [6].) Then $\tilde{G}$ is a separated Borel space. Indeed, every point of $\widetilde{G}$ is a Borel set.

Proposition 3. The central decomposition of a representation $L$ of a separable locally compact group $G$ may always be taken over the Borel space $\tilde{G}$. More explicitly, there exists a $\sigma$-finite measure $\mu$ on $\tilde{G}$ and $\mu$-measurable map $y \rightarrow L^{y}$ of $\widetilde{G}$ into $G^{c}$ such that $L^{v} \in y$ and $L \simeq \int_{G} L^{v} d \mu(y)$.

The method of proof is to begin with the central decomposition of $L$ over an arbitrary space $Z$, and then to transfer the corresponding measure over to $\widetilde{G}$, by means of a mapping of $Z$ into $\widetilde{G}$. Each measure $\mu$ determined on $\widetilde{G}$ by Proposition 3 is tame in the sense that $(\widetilde{G}, \mu)$ is measure space isomorphic to a $\sigma$-finite Borel measure on the unit interval $[0,1]$, with the usual Borel structure.

Proposition 4. The above decomposition theory determines a one-toone, lattice isomorphism of $Q$, the lattice of quasi-equivalence classes of representations of $G$, onto a lattice ideal of tame measure classes on $\tilde{G}$.

The image of $Q$ under this isomorphism is called the canonical measure lattice on $\widetilde{G}$, and is denoted $\operatorname{err}(\widetilde{G})$. If $G$ is a type I group, $\operatorname{ext}(\tilde{G})$ is the lattice of all tame measure classes on $\tilde{G}$. In general, however, $\operatorname{err}(\widetilde{G})$ is a proper lattice ideal of the lattice of all tame measure classes on $\tilde{G}$. We have in our possession a (somewhat superficial) characterization of $\operatorname{err}(\widetilde{G})$ which will be published elsewhere.

We next present a formulation of multiplicity theory which (a) meshes with the classification of quasi-equivalence classes given above, (b) distinguishes representations, up to unitary equivalence, within a quasi-equivalence class, and (c) casts the multiplicity theory for type I, II and III representations into the same mold.

Let $L$ be a quasi-equivalence class and $\mu$ the corresponding measure 
class on $\tilde{G}$. A multiplicity function for $\mu$ is a function $f$, defined on the lattice ideal of all Borel measure classes $\nu$ on $\widetilde{G}$ such that $\nu \leqq \mu$, having non-negative real numbers and $\infty$ for values, and satisfying the additional properties: (a) If $\nu \equiv 0$, then $f(\nu)=0$, (b) if $\nu_{1}$ and $\nu_{2}$ are Borel measure classes on $\tilde{G}$ such that $0 \neq \nu_{1} \leqq \nu_{2} \leqq \mu$, then $f\left(\nu_{2}\right) \leqq f\left(\nu_{1}\right)$ and (c) if $\nu$ is in the domain of $f$ and is the supremum of a countable family $\left\{\nu_{j}\right\}$ of two-by-two disjoint, nonzero measure classes on $\tilde{G}$, then $f(\nu)=\inf \left\{f\left(\nu_{j}\right)\right\}$.

Every $L$ in $Q$ may be expressed in the form $L=L_{\mathrm{I}} \vee L_{\mathrm{II}} \vee L_{\mathrm{III}}$, where $L_{i}$ is a type $i$ element of $Q, i=\mathrm{I}, \mathrm{II}$, III [5, Theorem 1.2]. $L_{\mathrm{II}}$ is called the type II part of $L$. A representation is infinite if $L \simeq \infty L$. A representation is finite if no subrepresentation is infinite.

Proposition 5. Suppose $L \in Q$ and $\mu$ is the corresponding measure class on $\tilde{G}$, given by Proposition 4. Let $M$ be any finite representation in the type II part of $L$. Then to each representation $L_{1} \in L$, there corresponds a multiplicity function $f_{1}$ for $\mu$. Two representations in the class $L$ are unitary equivalent if and only if they correspond to the same multiplicity function for $\mu$. (The definition of these functions depends on the choice of $M$.)

The multiplicity function corresponding to $L_{1} \in L$ is defined by considering the multiplicities of the primary components appearing in the central decomposition of $L_{1}$. These multiplicity functions describe the lattice structure within a quasi-equivalence class, in the following sense. If $f_{1}, f_{2}$ correspond to $L_{1}, L_{2}$ respectively, then $L_{1} \leqq L_{2}$ if and only if $f_{1}(\nu) \leqq f_{2}(\nu)$ for all $\nu \leqq \mu$. If $L$ is type I, the multiplicity theory is "absolute" in the sense that it does not depend on the choice of a finite type II representation $M$. In the type I case, the range of the multiplicity functions is the set of non-negative integers and $\infty$.

If $G$ is a type I group, the theory described above is essentially equivalent to that described in [6]. Indeed, the dual $\hat{G}$ may be mapped, one-to-one, onto the quasi-dual $\tilde{G}$ by sending an irreducible representation into the primary class containing it. This mapping, and its inverse, are both measurable. Under this identification, the order-preserving classification of quasi-equivalence classes by measure classes on $\widetilde{G}$ is equivalent to the order-preserving classification of multiplicity free representations by measure classes on $\hat{G}$. The theorem [5, Theorem 1.4] that every type $I$ representation is the direct sum of mutually disjoint representations of uniform multiplicity follows from the multiplicity theory described above and well known properties of multiplicity functions.

A completely analogous decomposition theory holds for *-repre- 
sentations (over separable complex Hilbert space) of separable Ba-

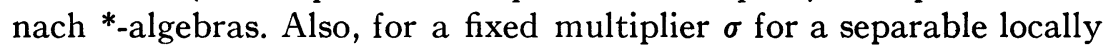
compact group $G$, we obtain a completely analogous decomposition theory for (projective) $\sigma$-representations of $G$. (Cf. [7].)

\section{BIBLIOGRAPHY}

1. J. Dixmier, Les algèbres d'opérateurs dans l'espace Hilbertien, Paris, GauthierVillars, 1957.

2. J. M. G. Fell, The dual spaces of $C^{*}$-algebras, Trans. Amer. Math. Soc. vol. 94 (1960) pp. 365-403.

3. A. Guichardet, Sur un problème posé par G. W. Mackey, C. R. Acad. Sci. Paris vol. 250 (1960) pp. 962-963.

4. G. W. Mackey, On induced representations of groups. Amer. J. Math. vol. 73 (1951) pp. 576-592.

5. - Induced representations of locally compact groups, II. The Frobenius reciprocity theorem, Ann. of Math. vol. 58 (1953) pp. 193-221.

6. - Borel structure in groups and their duals, Trans. Amer. Math. Soc. vol. 85 (1957) pp. 134-165.

7. - Unitary representations of group extensions. I, Acta Math. vol. 99 (1958) pp. 265-311.

8. F. I. Mautner, Unitary representations of locally compact groups. I, Ann. of Math. vol. 51 (1950) pp. 1-25.

9. M. A. Naimark, Factor-representations of a locally compact group, Soviet Math. Dokl. vol. 1 (1960) pp. 1064-1066.

10. J. von Neumann, On rings of operators. Reduction theory, Ann. of Math. vol. 50 (1949) pp. 401-485.

11. I. E. Segal, Decompositions of operator algebras. I and II, Memoirs Amer. Math. Soc., no. 9, 1951.

The Institute for Advanced Study 